Supplement of SOIL, 6, 523-539, 2020

https://doi.org/10.5194/soil-6-523-2020-supplement

(C) Author(s) 2020. This work is distributed under

the Creative Commons Attribution 4.0 License.

(c) (1)

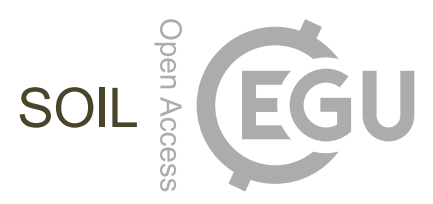

Supplement of

\title{
Nitrogen availability determines the long-term impact of land use change on soil carbon stocks in grasslands of southern Ghana
}

John Kormla Nyameasem et al.

Correspondence to: John Kormla Nyameasem (jnyameasem@gfo.uni-kiel.de, jnyameasem@gmail.com)

The copyright of individual parts of the supplement might differ from the CC BY 4.0 License. 


\section{Cut-use fodder grasses}

Andropogon gabonensis

Andropogon gayanus

Andropogon tectorum

Brachiaria brizantha

Brachiaria mutica

Brachiaria ruziziensis

Buchloe dactyloides

Cenchrus ciliaris

Cenchrus setigerus

Cut-use fodder herbs

\section{Calopogonium mucunoides}

Centrosema pubescens

Centrosema pubescens (ILCA)

\section{Desmodium distortum \\ Lablab purpureus}

Baphia nitida

Cajanus cajan

Cratylia argentea

Native grassland

Acacia spp.

Panicum decumbens

$\underline{\text { Seeded grazing fields }}$

Brachiaria brizantha

Food crop fields

Manihot esculenta
Clitoria ternatea

Desmanthus virgatus

Gliricidia sepium

Azadirachta indica

Talinum triangulare

\section{Cenchrus setigerus}

mopon giganteus

Cynodon plectostachyus

a decumbens

Digitaria swanzilandensis

Digitaria valida

Brachiaria ruziziensis

\section{Eragrostis curvula}

Eragrostis turgida

Heteropogon contortus

Hydropogon canaliculata

Hyperthelia dissoluta

Panicum maximum (Guinea fine)

Panicum maximum (ILCA)

Panicum maximum (local)

Panicum maximum (South Africa)

Macroptilium atropurpureum (Siratro) Macroptilium lathyroides

Mucuna pruriens

Leacaena lecocephala (Peru)

Leucaena leococephla (Langerham)
Paspalum conjugatum

Paspalum dilatatum

Paspalum notatum

Paspalum virgatum

Pennisetum purpureum

Pennisetum ruppelii

Seteria sp.

Seteria sphacelata

Sporobolus pyramidalis

Mucuna pruriens (Bunso

Pueraria phaseoloides

Sesbania grandiflora

Leucaena leococephla (local) Pithecelobium dulce

Moringa oleifera

Spondias mombin

Tripsacum laxum

Urochloa bolbodes

Urochloa pullulans

Vetiveria fulvivanus

Vetiveria zizanioides

Stylosanthes hamata
Stenotaphrum dimidiatum

Stylosanthes guianensis

\section{Cyperus rotundata}

Elaeis guineensis
Panicum maximum

${ }^{\mathrm{c}}$ Names in parenthesis indicate cultivar name

Digitaria decumbens 